\title{
Bioimpedancia eléctrica. Diferentes métodos de evaluación del estado nutricional en un centro periférico de hemodiálisis
}

Premio Sorin-Bellco de investigación en técnicas de diálisis

Cristina Mendías Benítez ${ }^{1}$ - Laura Alonso de Porras ${ }^{2}$ - Javier Barcia García ${ }^{1}$ - José Manuel Sánchez Oliva ${ }^{1}$ Elena Jiménez Quintana ${ }^{1}$ - Alfonso Lara Ruiz ${ }^{3}$ - Juan Chaín de la Bastida ${ }^{4}$

${ }^{1}$ Enfermeros del Centro de Hemodiálisis del Aljarafe. Sevilla

${ }^{2}$ Enfermera del Centro de Asenefro. Montequinto. Sevilla

${ }^{3}$ Director Médico del Centro Hemodiálisis Aljarafe. Sevilla

${ }^{4}$ Enfermero del Hospital Virgen del Rocío. Sevilla

\section{RESUMEN}

Introducción. Gracias a la bioimpedancia es posible valorar el estado de hidratación del paciente independientemente del peso corporal. También identifica las reservas proteicas/magras y reservas grasas en el paciente.

Objetivos. 1. Descripción del estado nutricional de la población estable en un centro periférico medido por: bioimpedancia, antropometría, valoración global subjetiva y datos analíticos. 2. Comparación y análisis de los diferentes métodos de valoración nutricional.

Metodología. Estudio analítico, prospectivo, comparativo de diferentes formas de valorar el estado

\begin{tabular}{|c|}
\hline Correspondencia: \\
Cristina Mendías Benítez \\
París, 32 \\
Villanueva del Ariscal \\
41808. Sevilla \\
mendiascris@hotmail.com
\end{tabular}

nutricional de los 52 pacientes estables de nuestro centro. Estudiamos la concordancia entre variables y comparamos parámetros significativos de cada método de valoración nutricional:

Resultados. Por valoración global subjetiva encontramos alto riesgo de desnutrición en un $63,46 \%$; de los pacientes, la bioimpedancia indica una malnutrición de un 59,61\%. La albúmina sérica muestra un $56,8 \%$ de los pacientes con un estado nutricional adecuado, mientras que están en este estado un $51,21 \%$ si se considera la transferrina sérica. Existen muchos métodos para valorar el estado nutricional de nuestros pacientes. Se observa correlación entre la valoración global subjetiva y la analítica nutricional.

\section{PALABRAS CLAVE:}

- BIOIMPEDANCIA ELÉCTRICA

- HEMODIÁLISIS

- ANTROPOMETRÍA

- ESTADO NUTRICIONAL 


\section{Electrical bioimpedance. Different methods of assessment of the nutritional state in a pe- ripheral haemodialysis centre}

\section{Abstract}

Introduction. Thanks to bioimpedance it is possible to evaluate the state of hydration of the patient independently of body weight. It also identifies the lean protein reserves and fat reserves in the patient.

Objectives. 1. Description of the nutritional state of the stable population in a peripheral centre measured by: bioimpedance, anthropometrics, subjective global assessment and analytical data. 2 . Comparison and analysis of the different nutritional evaluation methods.

Methodology. Analytical, prospective, comparative study of the different ways of evaluating the nutritional state of the 52 stable patients in our centre. We studied the match between variables and we compared significant parameters of each nutritional evaluation method:

Results. By subjective global assessment we found a high risk of malnutrition in $63.46 \%$ of patients, bioimpedance indicated malnutrition in $59.61 \%$. Serum albumin showed $56.8 \%$ of patients in an adequate nutritional state while $51.21 \%$ are in this state if serum transferrin is considered. There are many methods for evaluating the nutritional state of our patients. A correlation is observed between the subjective global assessment and the nutritional analysis.

\section{KEY WORDS}

- ELECTRICAL BIOIMPEDANCE

- HAEMODIALYSIS

- ANTHROPOMETRICS

- NUTRITIONAL STATE

\section{Introducción}

La nutrición se considera como un marcador de pronóstico fundamental de los pacientes con insuficiencia renal crónica y puede estar condicionada por múltiples factores negativos como la anorexia urémi- ca, las restricciones dietéticas, las disfunciones gastrointestinales, la fatiga postdialítica, o el hipercatabolismo dialítico. La desnutrición en el paciente renal crónico no solo disminuye la calidad de vida sino que también aumenta la morbimortalidad ${ }^{1}$. Todo ello indica que es conveniente evaluar periódicamente el estado nutricional de estos enfermos, así como incorporar en nuestra rutina como enfermeras de diálisis herramientas eficaces, sencillas y precisas para la determinación de la composición corporal.

Gracias a la bioimpedancia (BIA) posible obtener una valoración semicuantitativa, en escala de percentiles, del estado de hidratación del paciente en cualquier situación clínica e independientemente del peso corporal. Además, también identifica las reservas proteicas/magras $(F F M)$ y reservas grasas $(F M)^{2}$. Es una técnica no invasiva, capaz de valorar variaciones de hidratación, tan importantes en hemodiálisis para evitar complicaciones por sobrecarga de líquidos/ajuste del peso seco. Es un método barato, de simple ejecución (se puede realizar dentro de la misma unidad de diálisis), con escaso entrenamiento por diferentes observadores, y por tanto reproducible.

El fundamento de la BIA se basa en el principio de la impedancia (Z), mediante la cual se estudia la respuesta de un cilindro lleno de líquido al paso de la corriente eléctrica. Las cargas eléctricas de los iones presentes en el fluido se oponen al paso de la corriente eléctrica, y eso se conoce como Resistencia (R) expresada en $0 \mathrm{hm}^{2}$. La resistencia medida a través de un cilindro se relaciona directamente con la longitud del mismo ( $L$ ) e inversamente a su diámetro.

El cuerpo humano se puede conceptuar como un cilindro cuya longitud es la altura y su diámetro constante. La impedancia de un cuerpo humano es función de la resistividad específica del tejido "no lipídico", de su sección y de su longitud. La resistencia medida a través del cuerpo corregida para la altura refleja el volumen corporal. La relación entre la impedancia y resistencia se expresa por la ecuación $Z=R^{2}+X c^{2}$, donde $X_{c}$ es la reactancia, (que constituye la capacitancia de las membranas, expresada en $0 \mathrm{hm}^{2}$ ). La tangente entre R y Xc se conoce como Ángulo de Fase, que es un excelente marcador nutricional ${ }^{2}$. Este valor puede facilitar el seguimiento de ese estado en 
los pacientes con tratamiento renal, sometidos de forma frecuente a restricciones dietéticas.

Para la ejecución del análisis de BIA, el paciente se coloca en posición horizontal por lo menos 5 a 10 minutos, para distribuir de forma homogénea los fluidos corporales, con las piernas separadas aproximadamente $45^{\circ}$ y brazos separados del cuerpo $30^{\circ}$.

\section{Objetivos}

1. Describir el estado nutricional de la población estable de pacientes en hemodiálisis, en un centro periférico medido por BIA, antropometría, valoración global subjetiva (VGS) y datos analíticos.

2. Comparar y analizar diferentes métodos de valoración nutricional.

\section{Material y Métodos}

Estudio analítico, prospectivo, comparativo de diferentes formas de valorar el estado nutricional de los pacientes de nuestro centro, mediante antropometría, VGS, BIA y analítica nutricional.

La población inicial de estudio fueron 60 pacientes (41 hombres, 19 mujeres) considerados "estables", según los criterios del estudio nutricional multicéntrico de nuestra provincia del año 20043, de un total de 95 pacientes que componían nuestro centro en octubre del 2006. En el transcurso del estudio (octubre 2006 a enero 2007) abandonan el estudio varios pacientes ( 1 por trasplante, 5 por no querer participar en el estudio, 1 por problemas en el acceso vascular y 1 por ingreso hospitalario), quedando un total de 52 pacientes estables (36 hombres y 16 mujeres), con edades entre 20-87 años.

El paquete estadístico utilizado fue el SPSS 13.0. Estudiamos la concordancia entre variables, mediante el coeficiente de correlación de Spearman. Se comparan determinados parámetros significativos de cada método de valoración nutricional:

- Las reservas grasas obtenidas por BIA con la analítica nutricional (FM vs colesterol y FM vs triglicéridos) y con antropometría [FM vs pliegue cutáneo tricipital (PCT)].
- Las reservas magras obtenidas por BIA con analítica nutricional (FFM vs albúmina) y con antropometría [FFM vs circunferencia braquial (CB)].

- El estado nutricional obtenido por BIA con la valoración global subjetiva [índice de masa celular (BCMI) vs VGS e índice de masa corporal (BCM) vs VGS] y con analítica nutricional [ángulo de fase (AF) vs albúmina, AF vs PCR].

El aparato utilizado para analizar la BIA es el BIA101 Aker ${ }^{\circledR}$ (figura 1), un analizador por impedancia para el análisis de la composición corporal. El análisis impedanciométrico con BIA $101 \AA$ se obtuvo con la técnica clásica tetrapolar con corriente sinusoidal a $50 \mathrm{KHz}$ de frecuencia. El valor de la corriente se mantuvo constante en 800 microamperios sobre cargas de 1 a $4000 \Omega$. Para saturar el organismo con la corriente, los inyectores se posicionaron distalmente en la línea metacarpal y metatarsal, a través de electrodos de superficie. Los sensores se unieron a través de electrodos a las articulaciones de la muñeca y del tobillo, y por lo tanto en el interior del campo eléctrico (figura 2). La medida se realizó

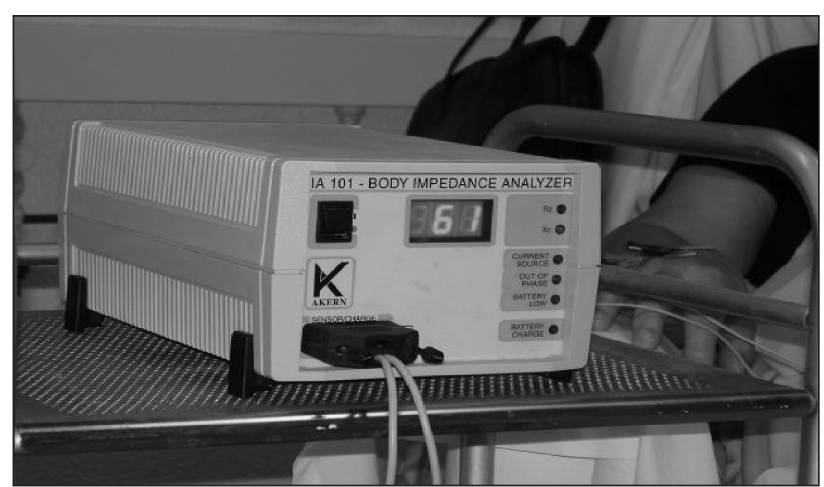

Figura 1: Monitor de bioempedancia

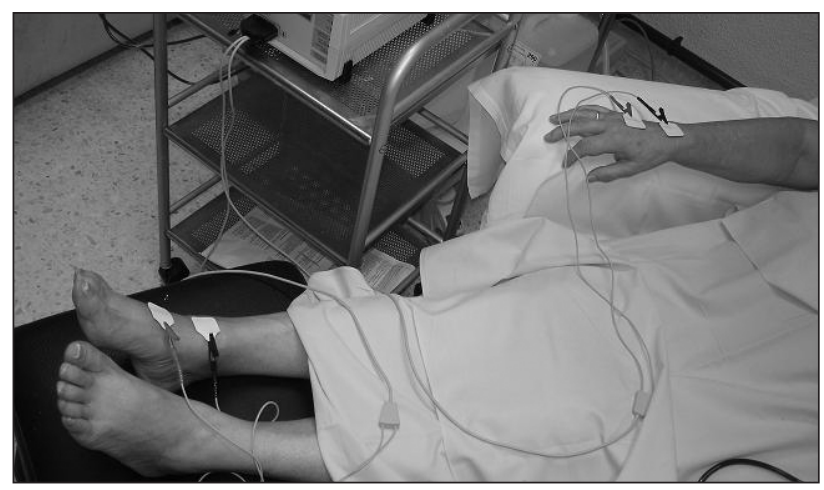

Figura 2: Determinación de la bioempedancia 
el día del medio de la semana (miércoles o jueves) antes de la conexión y post hemodiálisis.

Los datos obtenidos se analizaron mediante el programa Bodygram para Windows 95-98, con un software que analiza la composición corporal interpretando los datos de resistencia, reactancia, peso seco, talla, sexo y edad.

Las medidas antropométricas (talla, peso seco, CB, PCT, PCS) se realizaron al término de la sesión de hemodiálisis de mitad de semana, siguiendo las recomendaciones de las guías D0QUI de junio del 2000 sobre el perfeccionamiento de las mediadas antropométricas, con valores de referencia para los pliegues cutáneos del percentil 50 de las tablas adaptadas de Durnin JVGA ${ }^{4}$. Se utilizaron cintas métricas inextensibles y con plicómetros (lipocalipers Ross Adipometer $\left.{ }^{\circledR}\right)$ usados habitualmente en la Unidad de Dietética del hospital de nuestra provincia, siendo de precisión contrastada y validada.

Para la VGS utilizamos el mismo plicómetro, y el cuestionario usado en el estudio multicéntrico nutricional de la provincia del año 2004². Todos los pacientes fueron tallados y pesados descalzos, con ropa liviana y en posición antropométrica o postura estándar erecta.

Para evitar sesgos en las medidas antropométricas y VGS se instruyeron a 3 enfermeros, evitando posibles "problemas por el observador". Para las medidas de la BIA si participaron los demás enfermeros que componen el estudio. Todas las medidas antropométricas se realizaron por triplicado y se calculó la media, realizándose postdiálisis.

\section{Resultados}

La correlación obtenida por Spearman's rho de los parámetros estudiados fue la siguiente:

- FM vs colesterol $=0,327(r: 0,05)$.

- FM vs trigliceridos =0,317 ( $r: 0,05)$.

- FM vs PCT =0,446 ( $r: 0,01)$.

- FFM vs albúmina $=0,521$ ( $r: 0,01)$.

- FFM vs $C B=0,254$ is not significant $(0,069)$.

- BCMI vs VGS = - 0,534 ( $r: 0,01)$.
- BCM vs VGS = - 0,539 ( $r: 0,01)$.

- AF vs albúmina $=0,418$ ( $r: 0,01)$.

- AF vs nPCR =0,380 ( $r: 0,01)$.

- El estado nutricional obtenido por VGS fue: un alto riesgo de desnutrición en un $63,46 \%$ de los pacientes, un $7,69 \%$ de nutrición normal y un 29,92\% de desnutrición leve (figura 3 ).

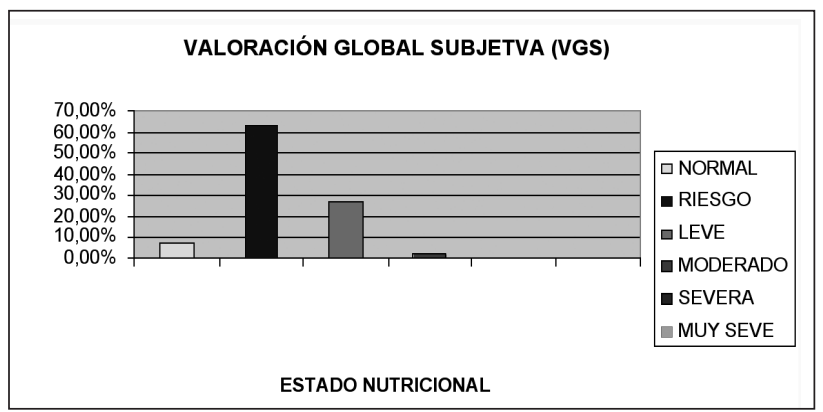

Figura 3: Estado nutricional según valoración global subjetiva

- El estado nutricional por BIA, valorando el índice de masa celular BCMI posthemodiálisis, indicó una malnutrición de un $59,61 \%$ de pacientes y un $40,38 \%$ de nutrición normal (figura 4).

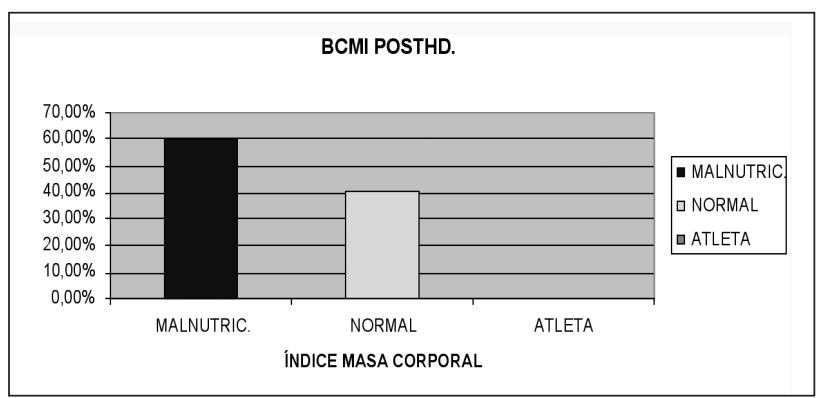

Figura 4: Estado nutricional según índice de masa celular

- La clasificación nutricional a partir de la albúmina sérica fue: un 56,8\% paciente con un estado nutricional adecuado, un $43,18 \%$ con desnutrición leve y ningún paciente con desnutrición moderada o grave (figura 5).

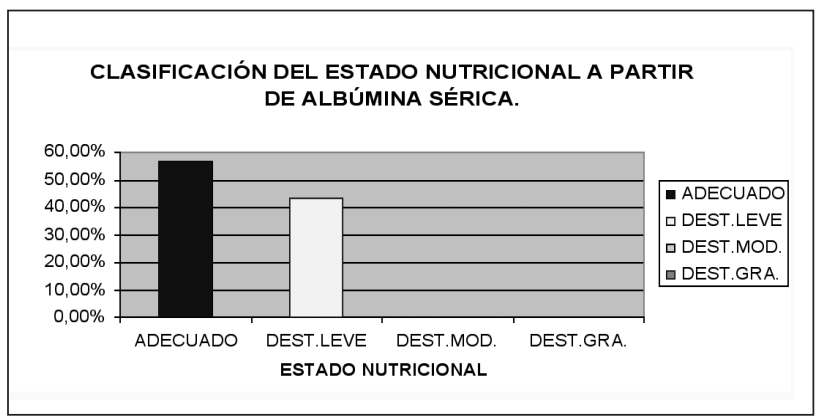

Figura 5: Estado nutricional según albúmina sérica 
- La clasificación del estado nutricional a partir de la transferrina sérica fue: un $51,21 \%$ de pacientes con un estado nutricional adecuado, un $31,70 \%$ con desnutrición leve, un $17,07 \%$ con desnutrición moderada y ningún paciente con desnutrición grave (figura 6).

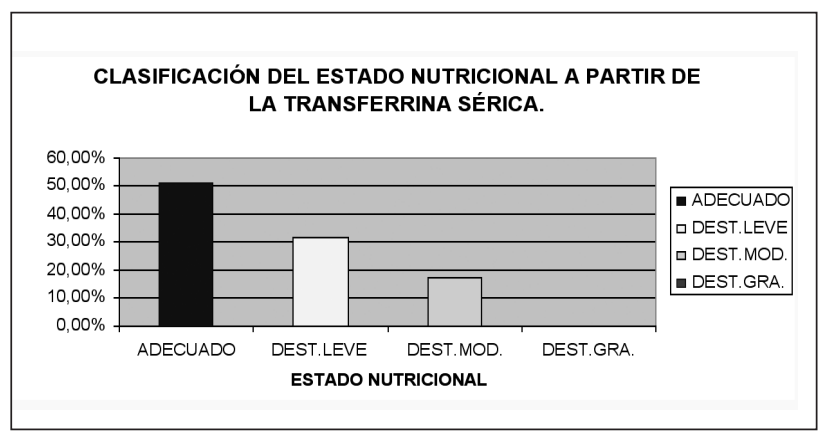

Figura 6: Estado nutricional según transferrina

\section{Discusión}

Existen muchos métodos para valorar el estado nutricional de nuestros pacientes, al utilizarlos y compararlos observamos una correlación entre la valoración global subjetiva y la analítica nutricional de los pacientes, pero no con la bioempedancia ya que esta es más general, y no encuadra a los pacientes en malnutridos, normales o atletas; es decir no hace clasificaciones intermedias como la valoración global subjetiva.
Por otra parte, las reservas calóricas, según antropometría, BIA y analítica nutricional si tienen buena correlación; en general, nuestros pacientes muestran unas reservas calóricas altas, con un número importante de pacientes obesos.

Por último considerar que el ángulo de fase obtenido mediante BIA es un buen marcador nutricional.

\section{Bibliografía}

1. Cigarrán S, Barril G, Vernis C. Evaluación del estado nutricional de los pacientes renales y ajuste del peso seco en CAPD y HD: papel de la Bioimpedancia. J. Biomed 2004; 1:16-23.

2. Morales R, Román AC. Composición corporal: intervalos de lo normal en el studio mediante bioimpedancia eléctrica de la población de referencia. Medisan 2004; 8(4):22-34.

3. Manzano JM. Valoración antropométrica de la población renal crónica estable en hemodiálisis en la provincia de Sevilla. Rev Soc Enfer Nefro 2006; 9(3): 218-225.

4. Piccoli A, Nescolarde LD, Rosell J. Análisis convencional y vectorial de la bioimpedancia en la práctica clínica. Nefrología 2002; 22 (3). 